\title{
Rumen bacterial community evaluated by 454 pyrosequencing and terminal restriction fragment length polymorphism analyses in dairy sheep fed marine algae
}

\author{
T. Castro-Carrera, ${ }^{*} \dagger$ P. G. Toral, ${ }^{*}$ P. Frutos, ${ }^{\star}$ N. R. McEwan,† G. Hervás, ${ }^{\star}$ L. Abecia,‡ E. Pinloche,† \\ S. E. Girdwood, $†$ and A. Belenguer*1 \\ *Instituto de Ganadería de Montaña, 24346 Grulleros, León, Spain \\ †Institute of Biological, Environmental and Rural Sciences, Aberystwyth University, Aberystwyth SY23 3DA, United Kingdom \\ łInstituto de Nutrición Animal, Estación Experimental del Zaidín, 18008 Granada, Spain
}

\begin{abstract}
Developing novel strategies to increase the content of bioactive unsaturated fatty acids (FA) in ruminantderived products requires a deeper understanding of rumen biohydrogenation and bacteria involved in this process. Although high-throughput pyrosequencing may allow for a great coverage of bacterial diversity, it has hardly been used to investigate the microbiology of ruminal FA metabolism. In this experiment, 454 pyrosequencing and a molecular fingerprinting technique (terminal restriction fragment length polymorphism; T-RFLP) were used concurrently to assess the effect of diet supplementation with marine algae (MA) on the rumen bacterial community of dairy sheep. Eleven lactating ewes were divided in 2 lots and offered a total mixed ration based on alfalfa hay and concentrate (40:60), supplemented with 0 (control) or 8 (MA) g of $\mathrm{MA} / \mathrm{kg}$ of dry matter. After $54 \mathrm{~d}$ on treatments, animals were slaughtered and samples of rumen content and fluid were collected separately for microbial analysis. Pyrosequencing yielded a greater coverage of bacterial diversity than T-RFLP and allowed the identification of low abundant populations. Conversely, both molecular approaches pointed to similar conclusions and showed that relevant changes due to MA addition were observed within the major ruminal phyla, namely Bacteroidetes, Firmicutes, and Proteobacteria. Decreases in the abundance of unclassified Bacteroidales, Porphyromonadaceae, and Ruminococcaceae and increases in as-yet uncultured species of the family Succinivibrionaceae, might be related to a potential role of these groups in different pathways of rumen FA metabolism. Diet supplementation with MA, however, had no effect on the relative abundance of Butyrivibrio and Pseudobutyrivibrio genera. In addition, results
\end{abstract}

Received July 9, 2013.

Accepted November 29, 2013.

${ }^{1}$ Corresponding author: a.belenguer@csic.es from both 454 pyrosequencing and T-RFLP indicate that the effect of MA was rather consistent in rumen content or fluid samples, despite inherent differences between these fractions in their bacterial composition. Key words: ewe, lipid, ruminal microbiota, $16 \mathrm{~S}$ rDNA

\section{INTRODUCTION}

Dietary PUFA are, in general, toxic for the rumen microbiota, although certain bacteria are able to metabolize and detoxify them through biohydrogenation (BH; Maia et al., 2007, 2010). This process results in the formation of some intermediates with bioactive characteristics (e.g., conjugated linoleic acid) that will then appear in ruminant-derived products (Lourenço et al., 2010). Recent in vivo investigations question some results from earlier in vitro assays suggesting that FA biohydrogenation is achieved only by a small group of bacteria. In fact, it is now accepted that the rumen microbial ecology of FA metabolism is much more complex, and most aspects on the response to dietary lipids are still uncertain (Boeckaert et al., 2008; Huws et al., 2011; Toral et al., 2012).

Molecular fingerprinting techniques, such as terminal restriction fragment length polymorphism (T-RFLP) or denaturing gradient gel electrophoresis (DGGE), are very useful to compare the structure and diversity of ruminal ecosystems and have allowed the detection of groups of bacteria, some of them as-yet uncultured or unclassified, with a potential predominant role in one or more metabolic pathways of $\mathrm{BH}$ in the rumen (Boeckaert et al., 2008; Belenguer et al., 2010; Huws et al., 2011). Nevertheless, they offer restricted information on less abundant taxa, which may bias the results. Thus, for instance, a previous study in dairy sheep fed marine lipids (i.e., $8 \mathrm{~g}$ of marine algae $/ \mathrm{kg}$ of $\mathrm{DM}$ ) showed marked changes in the concentration of $\mathrm{BH}$ intermediates that were only accompanied by little variations in the abundance of ruminal bacteria related to the BH process (Toral et al., 2012). 
This constraint might perhaps be overcome with next-generation techniques, such as high-throughput pyrosequencing (Hall, 2007). Yet, despite the much greater coverage of the microbial diversity allowed by this method, pyrosequencing has been little used in the study of rumen microbial ecology (Callaway et al., 2010; de Menezes et al., 2011; Pope et al., 2012); to our knowledge, only one article is available on ruminants fed lipids, with nonlactating cows (Zened et al., 2013). Pyrosequencing, conversely, is a high cost and not always available methodology.

Therefore, the first aim of the current study was to assess the effect of diet supplementation with marine algae (MA) on the rumen bacterial community structure of lactating sheep using T-RFLP and 454 pyrosequencing approaches. In addition, as ruminal cannulation is not really feasible in lactating sheep and stomach probe is often used to collect rumen samples, which rely solely on the liquid fraction (Belenguer et al., 2010; Toral et al., 2012), a second objective was to investigate if samples of rumen fluid may be an alternative to those of whole rumen content to examine the effect of lipid supplementation on the bacterial community.

\section{MATERIALS AND METHODS}

\section{Animals, Diets, and Experimental Design}

All experimental procedures were performed in accordance with Spanish Royal Decree 1201/2005 for the protection of animals used for experimental and other scientific purposes.

Twelve lactating Assaf ewes were recruited for the experiment, but due to health issues, 1 animal was withdrawn. Therefore, 11 sheep $(82.4 \pm 3.26 \mathrm{~kg}$ of BW $)$ were randomly distributed in 2 lots, which in turn were assigned to 1 of 2 dietary treatments. Diets were prepared weekly and consisted of a TMR based on alfalfa hay and a concentrate (40:60) supplemented with 0 (control diet; $\mathrm{n}=5$ ) or $8 \mathrm{~g}$ of marine algae (DHA Gold Animal Feed Ingredient, Martek Biosciences Corp., Columbia, MD) per kilogram of DM (MA diet; $\mathrm{n}=$ 6 ). The concentrate was composed of corn grain, soybean meal, barley grain, beet pulp, a vitamin-mineral supplement, and molasses to reduce selection of dietary components. Diets also included $25 \mathrm{~g}$ of sunflower oil/ $\mathrm{kg}$ of DM (Carrefour S.A., Madrid, Spain) and were offered daily ad libitum at 0900 and $1900 \mathrm{~h}$ throughout the 54-d experiment (for details, see Bichi et al., 2013).

\section{Measurements, Sampling Procedures, and Analyses}

After $54 \mathrm{~d}$ of treatment, ewes were slaughtered humanely by an intravenous injection of a euthanasia drug (T-61; Intervet, Salamanca, Spain). The rumen was excised and carefully removed, then the rumen content (RC) was homogenized and sampled from each animal. After that, it was strained through 2 layers of muslin and an aliquot of the resulting supernatant, which represented the rumen fluid ( $\mathbf{R F})$, was taken. All samples were immediately frozen at $-80^{\circ} \mathrm{C}$, freeze-dried, and stored again at $-80^{\circ} \mathrm{C}$ until analysis.

\section{DNA Extraction and Quantification}

Freeze-dried rumen samples were thoroughly homogenized before DNA extraction, which was performed by physical disruption using a bead beater (Mini-bead Beater, BioSpec Products, Bartlesville, OK) and subsequent DNA purification with the QIAamp DNA Stool Mini Kit (Qiagen Inc., Valencia, CA), as reported in Belenguer et al. (2010). The DNA was extracted twice from each sample, and duplicates were combined and used as templates for T-RFLP and 454 pyrosequencing analyses. The DNA concentrations and purity were measured by spectrophotometry (NanoDrop ND-1000 Spectrophotometer; Nanodrop Technologies, Wilmington, DE).

\section{T-RFLP Analysis}

This analysis was performed as outlined previously (Belenguer et al., 2010), with a universal bacteriaspecific primer pair set $(27 \mathrm{f}=$ AGAGTTTGATCCTGGCTCAG; and 1389r = ACGGGCGGTGTGTACAAG; Hongoh et al., 2003) and 3 restriction enzymes (HhaI, MspI, and HaeIII). The lengths of the fluorescently labeled terminal restriction fragments (T-RF) were determined with the size standard ET-900-R (GE Healthcare Life Sciences, Buckinghamshire, UK) using the GeneMarker Analysis software (SoftGenetics, State College, PA). To infer the potential bacterial composition in the samples, in silico restriction for the major rumen bacteria were obtained from the Ribosomal Database Project II website (http://rdp.cme.msu.edu/ index.jsp; Cole et al., 2009). Data from T-RFLP (size, bp, and peak area for each T-RF) were analyzed for peak filtering and binning, as outlined by Abdo et al. (2006), and used to determine the relative abundance of each fragment over the total peak area, as well as the diversity indices (number of T-RF or richness, Shannon and Shannon evenness indices; Hill et al., 2003).

\section{Pyrosequencing and Sequence Analysis}

Amplification of the bacterial V1-V2 regions of the 16S rRNA gene by PCR was performed using the primers pair 27f and 357r (CTGCTGCCTYCCGTA; Liu et 
al., 2007) appended with 454 sequencing adaptors at the $5^{\prime}$ end. Polymerase chain reaction for each sample was performed in triplicate in a total volume of $25 \mu \mathrm{L}$ containing $10 \times$ PCR buffer, $10 \mathrm{~m} M$ deoxyribonucleotide triphosphate mix, $10 \mathrm{pmol} / \mu \mathrm{L}$ of forward and reverse primers, 1 U of FastStart Taq DNA Polymerase (FastStart High Fidelity PCR System; Roche, Brandford, $\mathrm{CT})$, and $1 \mu \mathrm{L}$ of DNA template. The amplification program consisted of an initial denaturation step at $95^{\circ} \mathrm{C}$ for $2 \mathrm{~min}, 25$ cycles of denaturation at $95^{\circ} \mathrm{C}$ for 30 $\mathrm{s}$, annealing at $60^{\circ} \mathrm{C}$ for $30 \mathrm{~s}$, elongation at $72^{\circ} \mathrm{C}$ for 45 $\mathrm{s}$, and a final extension step at $72^{\circ} \mathrm{C}$ for $7 \mathrm{~min}$. The size of the PCR product (330 bp) was then checked on $1 \%$ agarose gel electrophoresis. Following this, triplicates were purified and normalized by quantitative DNA binding (SequalPrep kit; Invitrogen, Carlsbad, CA), pooled together, cleaned up through ethanol precipitation, and finally purified with the short fragment removal method described by Roche using their GS FLX amplicon DNA preparation guide and AMPure beads. The purified PCR products were quantified by the Quant-iT PicoGreen dsDNA quantification kit (Invitrogen) and mixed in equimolar amounts to a $10^{7}-\mathrm{mol}-$ ecules $/ \mu \mathrm{L}$ sample. The amplicon pooled libraries were pyrosequenced on a 454 FLX Titanium sequencer (454 Life Sciences; Roche).

Data of $16 \mathrm{~S}$ rRNA sequences generated by pyrosequencing were processed as follows. The flowgram files were converted to FASTA DNA and quality score files on the 454 cluster and transferred onto a Linuxbased workstation running quantitative insights into microbial ecology (QIIME) per scripted modules and workflow scripts (Caporaso et al., 2010). Sequences were filtered to exclude those that did not match the primer sequence, were less than 150 nt in length, or contained either one or more ambiguous bases or a long homopolymer $(>6)$. Errors of amplicon pyrosequences were corrected through the Acacia software (Bragg et al., 2012).

Operational taxonomic units (OTU) were generated by aligning the reads to the GreenGenes database (DeSantis et al., 2006) and clustered at 97\% sequence identity using the PyNAST tool (Caporaso et al., 2010) and the UCLUST algorithm (Edgar, 2010), respectively. Taxonomic classification was assigned with the basic local alignment search tool (Altschul et al., 1990). Alpha diversity (i.e., diversity within a sample) indices (observed species, Chao, and Shannon) were generated with the QIIME pipeline, whereas $\beta$ diversity (i.e., diversity between groups of samples) was used to create principal coordinate analysis (PCoA) plots using unweighted and weighted UniFrac distances. The UniFrac phylogenetic method (Lozupone and Knight,
2005), which considers phylogenetic lineages and not just shared OTU, was used for community-level comparisons with the trees constructed during the OTU picking script.

\section{Statistical Analysis}

Hierarchical clustering analysis with Ward's method based on Bray-Curtis distances was performed with R-project software (www.r-project.org, version 2.13.1) to build dendrograms with relative abundance data derived from T-RFLP (T-RF) and pyrosequencing (OTU at the genus level). Using the same software, multivariate ANOVA (MANOVA) of the same data was conducted to assess the effect of diet (control and $\mathrm{MA}$ ), fraction ( $\mathrm{RC}$ and $\mathrm{RF}$ ), and their interaction on the whole rumen bacterial structure.

In addition, relative abundances of each $\mathrm{T}-\mathrm{RF}$ or OTU were analyzed by one-way ANOVA, employing the MIXED procedure of the SAS software package (version 9.3, SAS Institute Inc., Cary, NC). As some results did not satisfy the assumptions of normality, data were transformed to $\log _{10}(\mathrm{n}+\mathrm{c}$; c being a constant of the same order of magnitude as the variable) before the analysis. The statistical model included the fixed effects of diet, fraction, and their interaction, plus the random effect of animal. Means were separated through the pdiff option of the lsmeans statement of the MIXED procedure. Differences were declared significant at $P<$ 0.05 and considered a trend toward significance at $P<$ 0.10 . Least squares means are reported.

\section{RESULTS}

\section{Bacterial Community Analysis by T-RFLP}

Rumen bacterial T-RFLP analysis generated, on average, $64.8 \pm 2.71,65.7 \pm 9.05$, and $152.9 \pm 7.42$ fragments with the enzymes HhaI, MspI, and HaeIII, respectively. Hierarchical clustering analysis grouped the bacterial structure of $\mathrm{RC}$ and $\mathrm{RF}$ samples derived from the same sheep (Figure 1a). In the dendrogram, most MA samples appeared in separate clusters, but the segregation by diet was not clear. According to MANOVA results, although the bacterial composition differed between rumen fractions $(P<0.05)$, the effect of MA was stronger $(P<0.001)$ and no significant interaction between diet and fraction was found $(P>$ $0.10)$.

The diversity indices did not differ between diets in data derived from MspI and HaeIII enzymes (Table $1 ; P>0.10$ ), whereas the HhaI digestion indicated a negative effect of the MA diet (i.e., Shannon and 
Table 1. Diversity indices $(\mathrm{R}=$ richness; $\mathrm{H}=$ Shannon; $\mathrm{E}=$ Shannon evenness; OS = observed species; and Chao) of bacterial communities in rumen content $(\mathrm{RC})$ or fluid $(\mathrm{RF})$ samples of ewes fed the experimental $\operatorname{diets}^{1}$

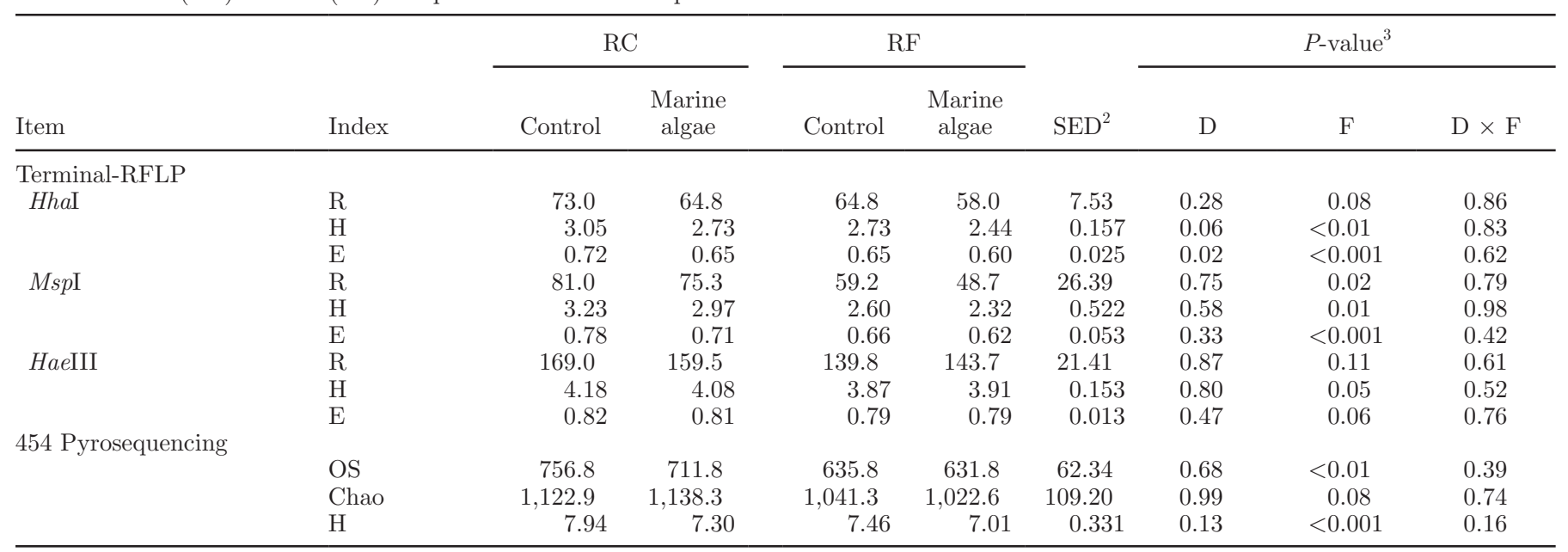

${ }^{1}$ Refers to a TMR containing 0 (control diet) or $8 \mathrm{~g}$ of marine algae (MA diet) per kilogram of DM.

${ }^{2} \mathrm{SED}=$ standard error of the difference.

${ }^{3}$ Probability of a significant effect due to diet (D), rumen fraction $(F)$, and their interaction $(\mathrm{D} \times \mathrm{F})$.

(a)

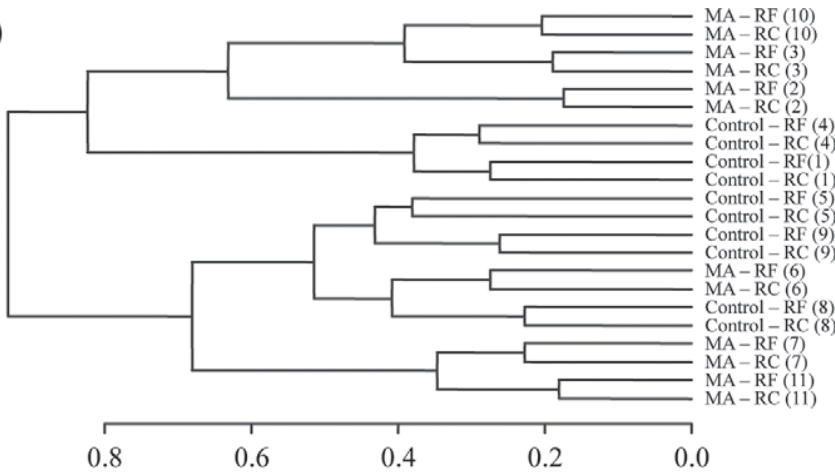

(b)

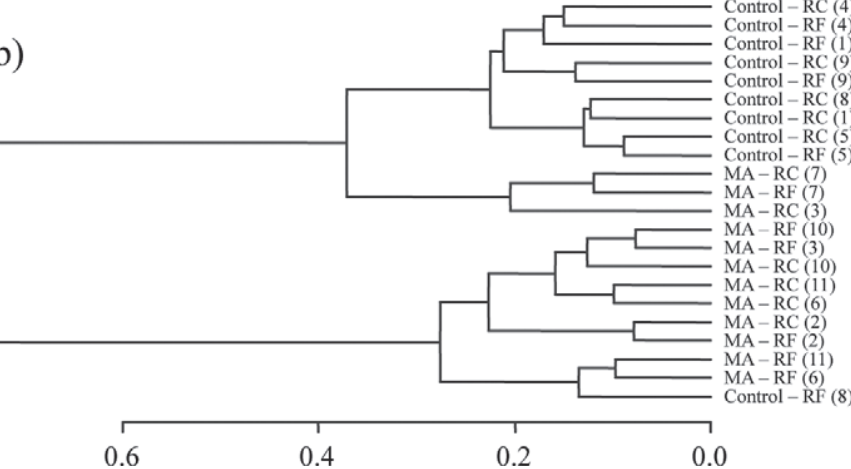

Figure 1. Cluster analysis of terminal-RFLP (a) or 454 pyrosequencing (b) profiles, based on Ward's method and the Bray-Curtis distances, of total bacteria in rumen content (RC) or fluid (RF) samples of ewes fed a diet with (marine algae $=\mathrm{MA}$ ) or without ( control) $8 \mathrm{~g}$ of marine algae $/ \mathrm{kg}$ of DM. Values in parentheses indicate the number of the animal.
Shannon evenness; $P<0.10)$ in both rumen fractions. All enzymes showed a greater bacterial diversity in $\mathrm{RC}$ compared with RF.

Putative taxonomic identification of major detected $\mathrm{T}-\mathrm{RF}$ revealed that they were likely to be derived from bacteria of the phylum Bacteroidetes (Table 2). Some of these fragments and others that may correspond with Succinivibrionaceae were more prevalent $(P<0.05)$ in $\mathrm{RF}$ than $\mathrm{RC}$, but this did not preclude from attributing similar effects to MA addition. For instance, both fractions showed that MA decreased T-RF that would match Porphyromonadaceae $(P<0.01)$ and Ruminococcaceae $(P<0.10)$, and increased $(P<0.01)$ others that may correspond to Succinivibrionaceae. Inconsistencies between fractions, such as the reduction in a T-RF putatively assigned to Bacteroidetes (98 bp with HhaI), which was only significant in RF samples, were rarely observed.

\section{Bacterial Community Analysis by Pyrosequencing}

An average of 5,688 sequences per sample were derived for bacterial diversity evaluation, with $4,945 \pm$ 253.5 used for analysis after quality control. Hierarchical clustering (Figure 1b) showed a certain separation by diet, but not by rumen fraction. The MANOVA results indicated that, although bacterial communities differed between RF and RC $(P<0.01)$, the effect of MA addition was stronger $(P<0.001)$, and no interaction was detected $(P>0.10)$. Similarly, PCoA showed no clear segregation due to the rumen fraction, whereas control samples were well separated from MA ones (Figure 2). 
Table 2. Relative frequencies [expressed as $\log _{10}(n+c)$ of percent over the total peak area, with original values in parentheses] of some terminal restriction fragments (T-RF) and their putative taxonomic identification in rumen content (RC) or fluid (RF) samples of ewes fed the experimental $\operatorname{diets}^{1}$

\begin{tabular}{|c|c|c|c|c|c|c|c|c|c|}
\hline \multirow[b]{2}{*}{$\begin{array}{l}\text { Taxonomic } \\
\text { identification }\end{array}$} & \multirow[b]{2}{*}{ T-RF (bp) } & \multicolumn{2}{|c|}{$\mathrm{RC}$} & \multicolumn{2}{|c|}{$\mathrm{RF}$} & \multirow[b]{2}{*}{$\mathrm{SED}^{2}$} & \multicolumn{3}{|c|}{$P$-value ${ }^{3}$} \\
\hline & & Control & Marine algae & Control & Marine algae & & $\mathrm{D}$ & $\mathrm{F}$ & $\mathrm{D} \times \mathrm{F}$ \\
\hline \multirow[t]{5}{*}{ Bacteroidetes } & 98 (HhaI) & $0.64^{\mathrm{b}}(4.67)$ & $0.58^{\mathrm{b}}(3.83)$ & $0.77^{\mathrm{a}}(6.20)$ & $0.51^{\mathrm{b}}(3.26)$ & 0.074 & 0.03 & 0.44 & 0.02 \\
\hline & $180(\mathrm{HhaI})$ & $0.57(2.06)$ & $0.49(1.34)$ & $0.66(2.83)$ & $0.49(1.35)$ & 0.079 & 0.11 & 0.16 & 0.18 \\
\hline & $91(M s p \mathrm{I})$ & $0.80(3.22)$ & $0.74(2.66)$ & $0.84(3.96)$ & $0.73(2.69)$ & 0.077 & 0.27 & 0.57 & 0.31 \\
\hline & $93(M s p \mathrm{I})$ & $0.56(1.62)$ & $0.67(2.88)$ & $0.58(1.69)$ & $0.61(2.30)$ & 0.095 & 0.46 & 0.59 & 0.28 \\
\hline & $263+264($ HaeIII $)$ & $1.32(20.95)$ & $1.36(23.63)$ & $1.38(24.15)$ & $1.42(26.59)$ & 0.047 & 0.22 & 0.07 & 0.98 \\
\hline \multirow[t]{2}{*}{ Porphyromonadaceae } & $84(\mathrm{MspI})$ & $0.33^{\mathrm{a}}(1.41)$ & $0.09^{\mathrm{b}}(0.40)$ & $0.36^{\mathrm{a}}(1.57)$ & $0.02^{\mathrm{c}}(0.24)$ & 0.078 & $<0.01$ & 0.39 & 0.04 \\
\hline & 257 (HaeIII) & $0.52(3.30)$ & $0.42(2.74)$ & $0.54(3.54)$ & $0.45(2.96)$ & 0.071 & 0.17 & 0.40 & 0.94 \\
\hline \multirow{3}{*}{ Ruminococcaceae } & $390(\mathrm{HhaI})$ & $-0.53(0.27)$ & $-0.89(0.00)$ & $-0.56(0.30)$ & $-0.89(0.00)$ & 0.169 & 0.07 & 0.64 & 0.64 \\
\hline & $320(\mathrm{Msp} \mathrm{I})$ & $0.76(4.04)$ & $0.41(0.84)$ & $0.64(2.65)$ & $0.36(0.47)$ & 0.078 & $<0.01$ & $<0.01$ & 0.22 \\
\hline & 280 (HaeIII) & $0.07(1.19)$ & $-0.15(0.78)$ & $0.02(1.55)$ & $-0.22(0.66)$ & 0.145 & 0.09 & 0.50 & 0.91 \\
\hline
\end{tabular}

${ }^{\mathrm{a}-\mathrm{c}}$ Within a row, different superscripts indicate significant differences $(P<0.05)$.

${ }^{1}$ Refers to a TMR containing 0 (control diet) or $8 \mathrm{~g}$ of marine algae (MA diet) per kilogram of DM.

${ }^{2} \mathrm{SED}=$ standard error of the difference.

${ }^{3}$ Probability of a significant effect due to diet $(\mathrm{D})$, rumen fraction $(\mathrm{F})$, and their interaction $(\mathrm{D} \times \mathrm{F})$.

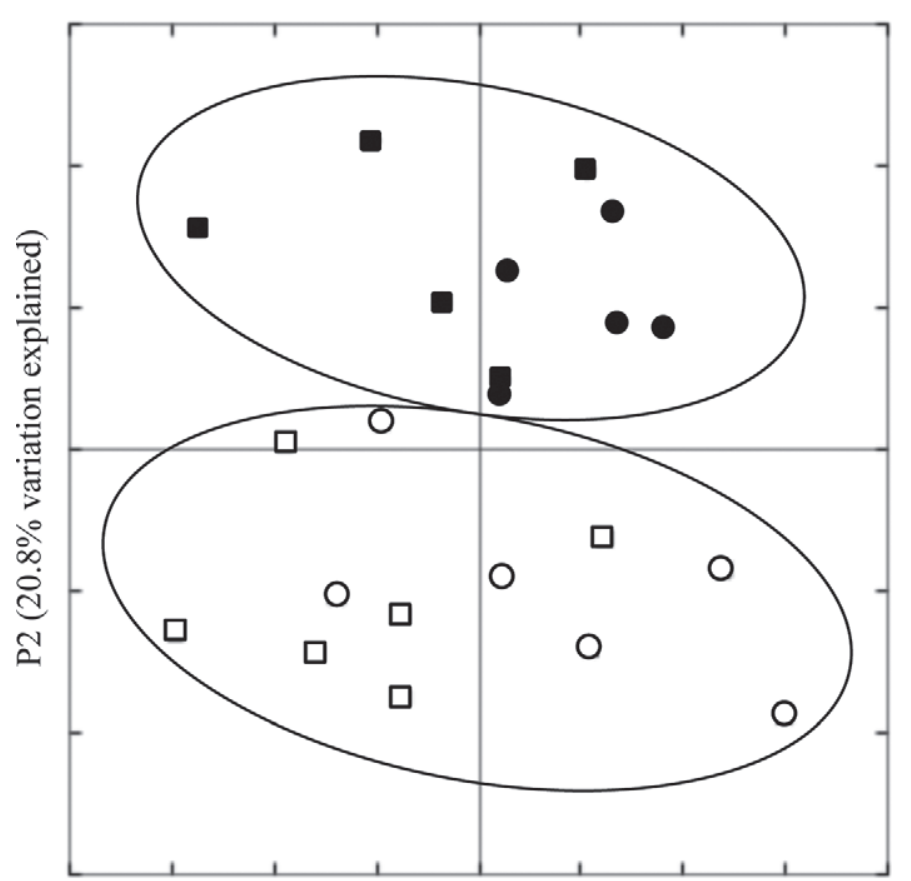

P1 (31.3\% variation explained)

Figure 2. Principal coordinate analysis plot of bacterial 16S rRNA gene sequences generated from 454 pyrosequencing run of DNA from rumen content (circles) or fluid (squares) samples of ewes fed a diet with $($ marine algae $=$ open symbols) or without $($ control $=$ closed symbols) $8 \mathrm{~g}$ of marine algae $/ \mathrm{kg}$ of DM. P1 and P2 = principal components 1 and 2 , respectively.
In terms of diversity (Table 1 ), the number of observed species was greater $(P<0.01)$ in $\mathrm{RC}$ samples and, consequently, some differences were also found in the Chao and Shannon diversity indices between RC and RF. Nonetheless, both fractions showed the same lack of significant effect of MA on diversity.

Regarding the bacterial community composition, Bacteroidetes was the dominant phylum (69-77\%; Figure 3), followed by Firmicutes (13-26\%) and Proteobacteria (1-7\%), whereas the remaining detected phyla (Fibrobacteres, Tenericutes, Cyanobacteria, Spirochaetes, Synergistetes, TM7, Elusimicrobia, SR1, Verrucomicrobia, Fusobacteria, Chloroflexi, and Actinobacteria) appeared at percentages lower than 1.5 (Supplemental Table S1; http://dx.doi.org/10.3168/jds.2013-7243).

Within Bacteroidetes, the abundance of Prevotellaceae, whose detected sequences belonged only to the genus Prevotella, represented 49 to $61 \%$ (Table 3 and Supplemental Table S1; http://dx.doi.org/10.3168/ jds.2013-7243). Despite the greater prevalence of this phylum in RF than in RC, similar effects of MA were observed in both rumen fractions for all identified subpopulations. Most relevant effects of MA included increases in the genus Bacteroides $(P<0.01)$ and the family S24-7 $(P<0.05)$, and decreases in Paraprevotellaceae $(P<0.10)$, Porphyromonadaceae $(P<0.001)$, and other unclassified members of the order Bacteroidales $(P<0.01$; Table 3$)$.

Within Firmicutes, most sequences belonged to the order Clostridiales, and Lachnospiraceae, Ruminococ- 
1666

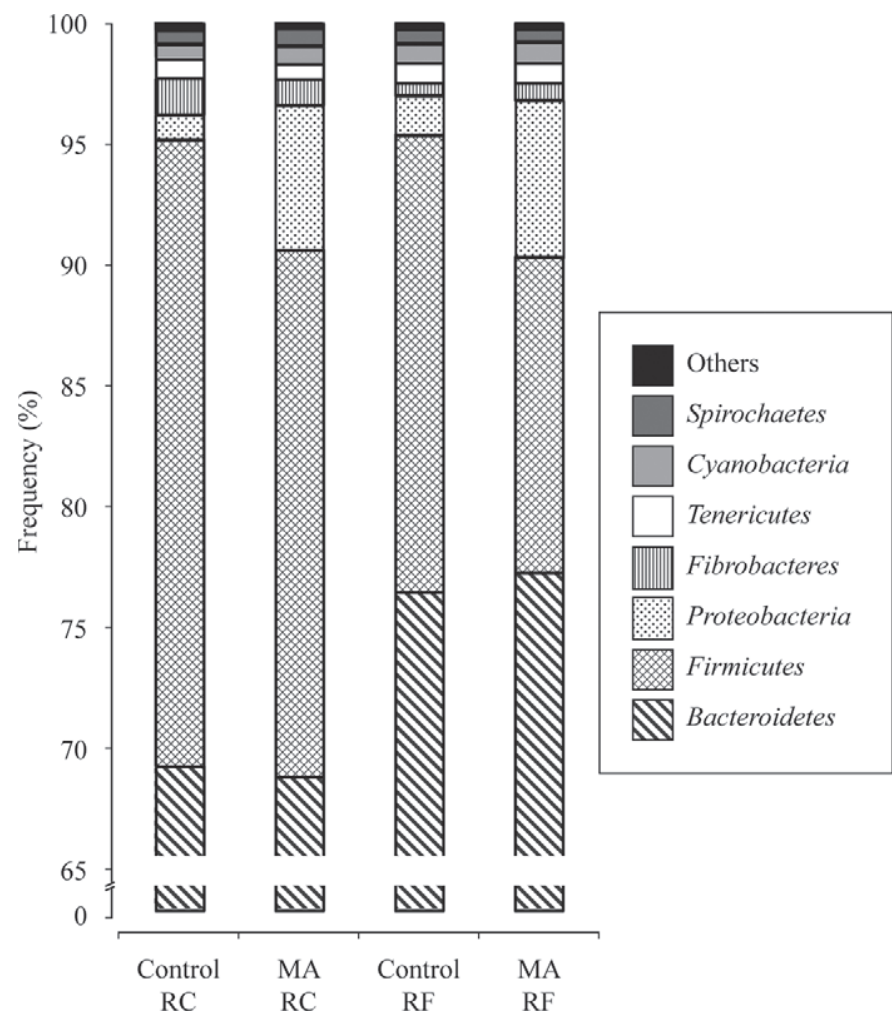

Figure 3. Relative abundances (\% of total sequences) of most abundant bacterial phyla identified by 454 pyrosequencing in rumen content (RC) or fluid (RF) samples of ewes fed a diet with (marine algae $=\mathrm{MA}$ ) or without (control diet) $8 \mathrm{~g}$ of marine algae $/ \mathrm{kg}$ of DM.

caceae, and Veillonellaceae were the most prevalent families. Again, although the relative abundance of Firmicutes was greater in RF, most changes due to MA addition were detected similarly in both fractions (e.g., the reduction of unclassified members of Ruminococcaceae and Clostridium; $P<0.05)$. Only bacterial groups of the family Veillonellaceae were affected differently by $\mathrm{MA}$ in RC and RF, with unclassified species showing a decrease just in the latter, and Selenomonas an increase in the former $(P<0.05$ and $P<0.10$, respectively, for diet $\times$ fraction). Regarding the phylum Proteobacteria, MA supplementation promoted, similarly in RC and $\mathrm{RF}$, at least a 4-fold increase in species of the family Succinivibrionaceae (Table 3 and Supplemental Table S1; http://dx.doi.org/10.3168/jds.2013-7243).

\section{DISCUSSION}

Developing novel strategies to increase the content of bioactive unsaturated FA in ruminant-derived products, such as milk and meat, requires a deeper understanding of ruminal $\mathrm{BH}$ and bacteria involved in this process (Boeckaert et al., 2009; Huws et al., 2011). Although pyrosequencing platforms may allow for a great cover- age of bacterial diversity (Hall, 2007), they have hardly been used to investigate the microbiology of rumen FA metabolism. Conversely, several reports in the literature demonstrate, through molecular fingerprinting techniques such as T-RFLP or DGGE, the complexity of the rumen ecosystem response to dietary lipid supplementation and the potential predominant role of as-yet uncultured bacteria in ruminal BH (Boeckaert et al., 2008; Huws et al., 2011; Toral et al., 2012). The choice of the type of methodology will depend on several factors, one of the most important being the compromise between the accuracy and precision of the results and the cost of the approach.

In the current study, both 454 pyrosequencing and TRFLP were used concurrently and the results pointed to similar conclusions, which may allow the usefulness of T-RFLP to be strengthened in this kind of study. Thus, hierarchical clustering, MANOVA, and PCoA analyses showed that, despite known variations in the bacterial composition of RC and RF (Pitta et al., 2010; de Menezes et al., 2011), dietary MA was stronger in shaping the bacterial community structure. This is in agreement with Pitta et al. (2010), who reported that the 2 rumen fractions studied (liquid and whole contents) were able to associate distinct bacterial communities with different forage diets. In the present study, samples were segregated in response to MA in spite of its low level of inclusion in the diet $(0.8 \%)$ and the small variations in the diversity indices estimated with either pyrosequencing or T-RFLP. These results are similar to those reported previously, using fingerprinting techniques, in sheep (Belenguer et al., 2010; Toral et al., 2012) and cattle (Boeckaert et al., 2007, 2008; Huws et al., 2011) fed marine lipids, and would underline the potent inhibitory effect of very long-chain PUFA on certain rumen bacteria (Maia et al., 2007, 2010).

The phylogenetic resolution of the 454 pyrosequencing allowed the identification at the phylum level of the entire set of sequences and up to the genus level of almost $75 \%$ (the remaining $25 \%$ was due to limitations in the available databases). Consistent with previous results in cattle, sheep, and goats (Stewart et al., 1997; Lee et al., 2012; Kittelmann et al., 2013), the phyla Bacteroidetes and Firmicutes were the most abundant in the rumen bacterial community, regardless of rumen fractions and effects of diet. Conversely, both $\mathrm{RF}$ and $\mathrm{RC}$ showed that the relative abundance of the phylum Proteobacteria was greater in ewes fed MA. These results agreed with detected T-RF, although pyrosequencing achieved the identification of 12 other phyla with much lower abundances $(<1.5 \%$ of total sequences; Supplemental Table S1; http:// dx.doi.org/10.3168/jds.2013-7243), some of which may be significant at a functional level and therefore 
Table 3. Relative abundances [expressed as $\log _{10}(n+c)$ of percent of total sequences, with original values in parentheses] of relevant orders, families, and genera identified by 454 pyrosequencing in rumen content (RC) or fluid (RF) samples of ewes fed the experimental diets ${ }^{1}$

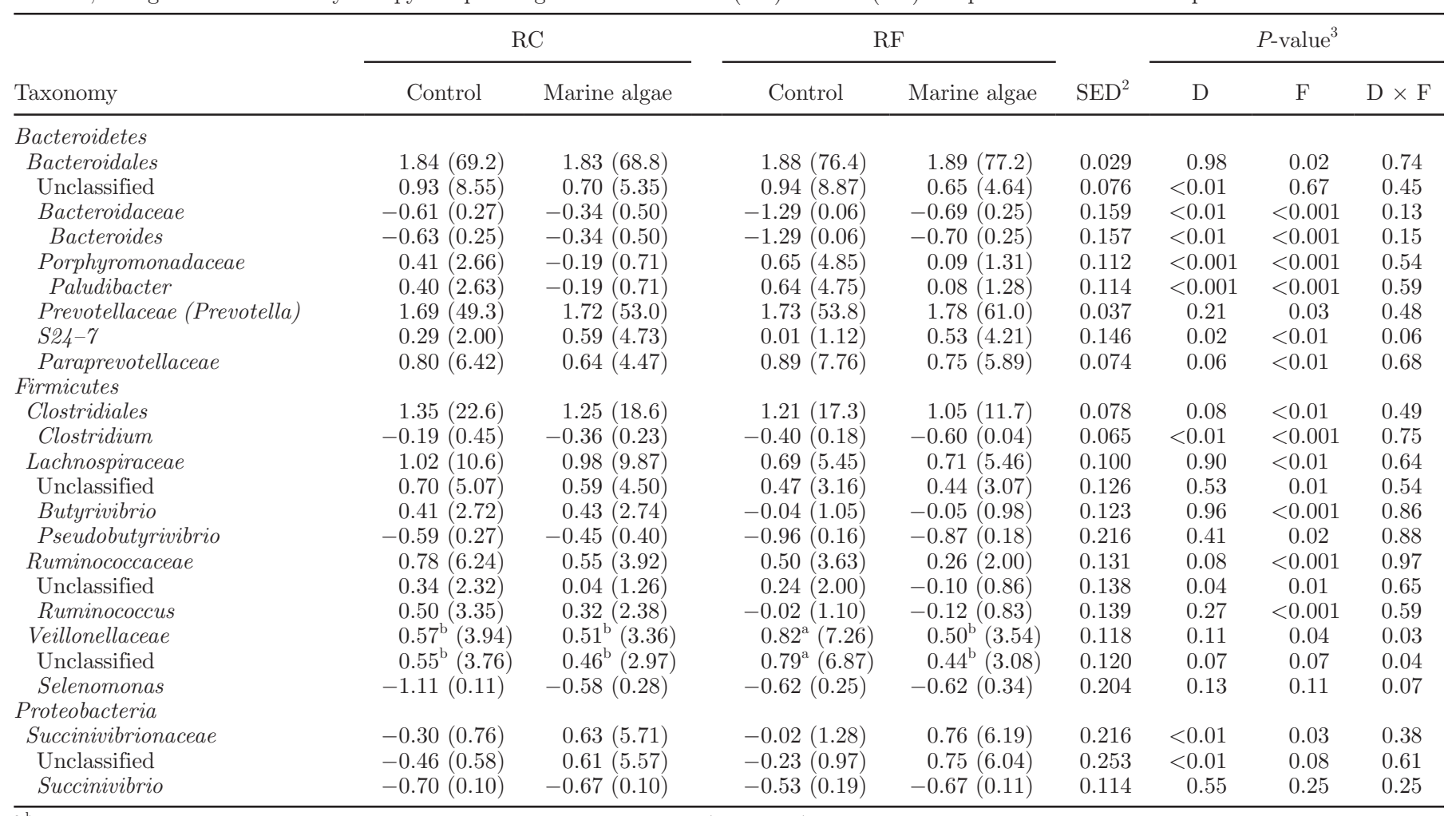

${ }^{a, b}$ Within a row, different superscripts indicate significant differences $(P<0.05)$.

${ }^{1}$ Refers to a TMR containing 0 (control diet) or $8 \mathrm{~g}$ of marine algae (MA diet) per kilogram of DM.

${ }^{2} \mathrm{SED}=$ standard error of the difference.

${ }^{3}$ Probability of a significant effect due to diet (D), rumen fraction $(\mathrm{F})$, and their interaction $(\mathrm{D} \times \mathrm{F})$.

important for the study (Stewart et al., 1997). However, MA supplementation had almost no effect on the relative abundance of those minor groups, with the exception of a reduction in the candidate phylum TM7, and all relevant changes were observed in Bacteroidetes, Firmicutes, and Proteobacteria phyla, in line with concomitant variations in T-RF.

Regardless of the diet and rumen fraction, the relative abundance of Bacteroidetes sequences (>68\%) were on the highest range reported for ruminants using both traditional (van Gylswyk, 1990) and molecular techniques (Lee et al., 2012; Pope et al., 2012; Wu et al., 2012). Within this phylum, the genus Prevotella represented more than $50 \%$ of total sequences in most samples (particularly in RF), which may suggest a relevant function in the rumen ecosystem. Its predominance agrees with previous studies (Stevenson and Weimer, 2007; Jami and Mizrahi, 2012; Wu et al., 2012) and is probably explained by the basal diet composition, with relatively low fiber content and supplemented with sunflower oil. In this regard, it is known that Prevotella degrades readily metabolizable carbohydrates (Stewart et al., 1997) and is more abundant in low-fiber diets (Pitta et al., 2010). In addition, several Prevotella strains have shown a low sensitivity to linoleic acid, the major FA in sunflower oil, both in vitro (Maia et al., 2007) and in vivo (Zened et al., 2013), as well as to very longchain PUFA (Maia et al., 2007; Huws et al., 2010), which is consistent with the lack of variation in ewes fed MA. However, the treatment with marine lipids had contrasting effects on other subpopulations within this large and diverse phylum, which suggests either different roles of these groups in $\mathrm{BH}$ or distinct sensitivities to the toxic effect of very long-chain PUFA.

Changes within Bacteroidetes due to MA, observed both with T-RFLP and pyrosequencing, included reductions in Porphyromonadaceae and relatively abundant bacteria identified as unclassified Bacteroidales. In this respect, and despite the limited knowledge on the relationship between Bacteroidetes members and dietary lipids (Maia et al., 2007; Huws et al., 2010), putative taxonomic identifications have linked unclassified Bacteroidales and Porphyromonadaceae strains with 18:0, trans-11, 18:1, and cis-9,trans-11 conjugated linoleic acid concentrations in the rumen of cattle fed fish oils (Huws et al., 2011). 
On the contrary, supplementing the dairy ewe diet with MA did not have an effect on the relative abundance of bacterial genera, including microorganisms that are by far the most active $\mathrm{BH}$ species isolated from the rumen, such as Butyrivibrio and Pseudobutyrivibrio (Paillard et al., 2007). These findings are in agreement with recent in vivo studies (Belenguer et al., 2010; Huws et al., 2011; Shingfield et al., 2012), which have suggested a lower relevance of those species in ruminal $\mathrm{BH}$ than initially thought. However, it must be considered that the functional activity of a microbial group may be affected without variations in its DNA abundance (Popova et al., 2011).

Other subpopulations of the phylum Firmicutes, such as unclassified Ruminococcaceae and Veillonellaceae strains and low abundant sequences corresponding to Selenomonas, were altered by MA. The increase in the relative abundance of the latter only tended to be significant in RC (Table 3) and, although it represented a very small fraction, the fact that it was present in all samples might indicate that Selenomonas occupies a special ecological niche in the rumen. In fact, and regarding rumen FA metabolism, the species Selenomonas ruminantium is known to be involved in FA hydration (Hudson et al., 1995), an alternative pathway that is enhanced in ewes fed marine lipids (Toral et al., 2012).

With regard to Ruminococcaceae, the relative abundance of unclassified species of this family was negatively affected by the treatment in both $\mathrm{RF}$ and $\mathrm{RC}$, which is consistent with a potential link with decreased ruminal 18:0 concentrations in animals fed MA (Boeckaert et al., 2008; Huws et al., 2011; Toral et al., 2012). Another example of the similar effect of the treatment on $\mathrm{RF}$ and $\mathrm{RC}$ was the notable increase in the relative abundance of the phylum Proteobacteria, particularly in sequences corresponding to unclassified Succinivibrionaceae, which is also in agreement with increases in 2 T-RF assigned phylogenetically to this family. Although Tokoyama and Davis (1970) showed a putative ability of Succinivibrio dextrinosolvens to biohydrogenate, and Li et al. (2012) pointed out a potential involvement of Proteobacteria in lipid metabolism, the role of these bacteria in $\mathrm{BH}$ is still uncertain. Despite the known differences in the bacterial composition of $\mathrm{RC}$ and $\mathrm{RF}$, which should serve as a note of caution when interpreting the effect of fat supplementation on the ruminal microbial community, the consistent effect of feeding MA on both fractions would suggest that, when rumen cannulation is not really feasible, fluid samples might be a valid alternative to infer the effect of dietary lipids on bacterial structure and diversity.

\section{CONCLUSIONS}

Pyrosequencing yielded a greater coverage of rumen bacterial diversity than T-RFLP and allowed the identification of low abundant populations. However, both molecular techniques show that the main changes due to MA addition are observed within the major ruminal groups, namely Bacteroidetes, Firmicutes, and Proteobacteria, and correspond in most cases to as-yet uncultured or unclassified bacteria. These results underline the complexity of the rumen ecosystem response to dietary lipid supplementation and the need for further research. In addition, both 454 pyrosequencing and TRFLP indicate that the effect of MA supplementation on bacterial diversity and structure is rather consistent in rumen content or fluid samples, despite inherent differences between these fractions in their microbial composition.

\section{ACKNOWLEDGMENTS}

This study was supported by the Spanish Ministry of Economy and Competitiveness (Madrid, Spain; AGL2011-23700). T. Castro-Carrera was granted a predoctoral fellowship from the Spanish National Research Council (CSIC, Madrid, Spain; JAE Programme) supported by the European Social Fund (European Commission, Brussels, Belgium). The authors gratefully acknowledge the research farm staff from the Instituto de Ganadería de Montaña for their help in the fieldwork.

\section{REFERENCES}

Abdo, Z., U. M. E. Schüette, S. J. Bent, C. J. Williams, L. J. Forney, and P. Joyce. 2006. Statistical methods for characterizing diversity of microbial communities by analysis of terminal restriction fragment length polymorphisms of $16 \mathrm{~S}$ rRNA genes. Environ. Microbiol. 8:929-938.

Altschul, S. F., W. Gish, W. Miller, E. W. Myers, and D. J. Lipman. 1990. Basic local alignment search tool. J. Mol. Biol. 215:403-410.

Belenguer, A., P. G. Toral, P. Frutos, and G. Hervás. 2010. Changes in the rumen bacterial community in response to sunflower oil and fish oil supplements in the diet of dairy sheep. J. Dairy Sci. 93:3275-3286.

Bichi, E., G. Hervás, P. G. Toral, J. J. Loor, and P. Frutos. 2013. Milk fat depression induced by dietary marine algae in dairy ewes: Persistency of milk fatty acid composition and animal performance responses. J. Dairy Sci. 96:524-532.

Boeckaert, C., V. Fievez, D. Van Hecke, W. Verstraete, and N. Boon. 2007. Changes in rumen biohydrogenation intermediates and ciliate protozoa diversity after algae supplementation to dairy cattle. Eur. J. Lipid Sci. Technol. 109:767-777.

Boeckaert, C., D. P. Morgavi, J. P. Jouany, L. Maignien, N. Boon, and V. Fievez. 2009. Role of the protozoan Isotricha prostoma, liquid-, and solid-associated bacteria in rumen biohydrogenation of linoleic acid. Animal 3:961-971.

Boeckaert, C., B. Vlaeminck, V. Fievez, L. Maignien, J. Dijkstra, and N. Boon. 2008. Accumulation of trans C-18:1 fatty acids in 
the rumen after dietary algal supplementation is associated with changes in the Butyrivibrio community. Appl. Environ. Microbiol. 74:6923-6930.

Bragg, L., G. Stone, M. Imelfort, P. Hugenholtz, and G. W. Tyson. 2012. Fast, accurate error-correction of amplicon pyrosequences using Acacia. Nat. Methods 9:425-426.

Callaway, T. R., S. E. Dowd, T. S. Edrington, R. C. Anderson, N. Krueger, N. Bauer, P. J. Kononoff, and D. J. Nisbet. 2010. Evaluation of bacterial diversity in the rumen and feces of cattle fed different levels of pyrosequencing dried distillers grains plus solubles using bacterial tag-encoded FLX amplicon pyrosequencing. J. Anim. Sci. 88:3977-3983.

Caporaso, J. G., J. Kuczynski, J. Stombaugh, K. Bittinger, F. D. Bushman, E. K. Costello, N. Fierer, A. G. Peña, J. K. Goodrich, J. I. Gordon, G. A. Huttley, S. T. Kelley, D. Knights, J. E. Koening, R. E. Ley, C. A. Lozupone, D. McDonald, B. D. Muegge, M. Pirrung, J. Reeder, J. R. Sevinsky, P. J. Turnbaugh, W. A. Walters, J. Widmann, T. Yatsunenko, J. Zaneveld, and R. Knight. 2010. QIIME allows analysis of high throughput community sequencing data. Nat. Methods 7:335-336.

Cole, J. R., Q. Wang, E. Cardenas, J. Fish, B. Chai, R. J. Farris, A. S. Kulam-Syed-Mohideen, D. M. McGarrell, T. Marsh, G. M. Garrity, and J. M. Tiedje. 2009. The ribosomal database project: Improved alignments and new tools for rRNA analysis. Nucleic Acids Res. 37:D141-145.

de Menezes, A. B., E. Lewis, M. O'Donovan, B. F. O'Neill, N. Clipson, and E. M. Doyle. 2011. Microbiome analysis of dairy cows fed pasture or total mixed ration diets. FEMS Microbiol. Ecol. 78:256-265.

DeSantis, T. Z., P. Hugenholtz, N. Larsen, M. Rojas, E. L. Brodie, K. Keller, and T. Huber. 2006. Greengenes, a chimera-checked 16S rRNA gene database and workbench compatible with ARB. Appl. Environ. Microbiol. 72:5069-5072.

Edgar, R. C. 2010. Search and clustering orders of magnitude faster than BLAST. Bioinformatics 26:2460-2461.

Hall, N. 2007. Advanced sequencing technologies and their wider impact in microbiology. J. Exp. Biol. 210:1518-1525.

Hill, T. C. J., K. A. Walsh, J. A. Harris, and B. F. Moffett. 2003. Using ecological diversity measures with bacterial communities. FEMS Microbiol. Ecol. 43:1-11.

Hongoh, Y., M. Yuzawa, M. Okhuma, and T. Kudo. 2003. Evaluation of primers and PCR conditions for the analysis of $16 \mathrm{~S}$ rRNA genes from a natural environment. FEMS Microbiol. Lett. 221:299-304.

Hudson, J. A., C. A. M. MacKenzie, and K. N. Joblin. 1995. Conversion of oleic acid to 10-hydroxystearic acid by two species of ruminal bacteria. Appl. Microbiol. Biotechnol. 44:1-6.

Huws, S. A., E. J. Kim, M. R. F. Lee, M. B. Scott, J. K. S. Tweed, E. Pinloche, R. J. Wallace, and N. D. Scollan. 2011. As yet uncultured bacteria phylogenetically classified as Prevotella, Lachnospiraceae incertae sedis and unclassified Bacteroidales, Clostridiales and Ruminococcaceae may play a predominant role in ruminal biohydrogenation. Environ. Microbiol. 13:1500-1512.

Huws, S. A., M. R. F. Lee, S. M. Muetzel, T. W. Scott, R. J. Wallace, and N. D. Scollan. 2010. Forage type and fish oil cause shifts in rumen bacterial diversity. FEMS Microbiol. Ecol. 73:396-407.

Jami, E., and I. Mizrahi. 2012. Composition and similarity of bovine rumen microbiota across individual animals. PLoS ONE 7:e33306.

Kittelmann, S., H. Seedorf, W. A. Walters, J. C. Clemente, R. Knight, J. I. Gordon, and P. H. Janssen. 2013. Simultaneous amplicon sequencing to explore co-occurrence patterns of bacterial, archaeal and eukaryotic microorganisms in rumen microbial communities. PLOS ONE 8:e47879.

Lee, H. J., J. Y. Jung, Y. K. Oh, S. S. Lee, E. L. Madsen, and C. O. Jeon. 2012. Comparative survey of rumen microbial communities and metabolites across one caprine and three bovine groups, using bar-coded pyrosequencing and ${ }^{1} \mathrm{H}$ nuclear magnetic resonance spectroscopy. Appl. Environ. Microbiol. 78:5983-5993.

Li, D., J. Q. Wang, and D. P. Bu. 2012. Ruminal microbe of biohydrogenation of trans-vaccenic acid to stearic acid in vitro. BMC Res. Notes 5:97.
Liu, Z., C. Lozupone, M. Hamady, F. D. Bushman, and R. Knight. 2007. Short pyrosequencing reads suffice for accurate microbial community analysis. Nucleic Acids Res. 35:e120.

Lourenço, M., E. Ramos-Morales, and R. J. Wallace. 2010. The role of microbes in rumen lipolysis and biohydrogenation and their manipulation. Animal 4:1008-1023.

Lozupone, C., and R. Knight. 2005. UniFrac: A new phylogenetic method for comparing microbial communities. Appl. Environ. Microbiol. 71:8228-8235.

Maia, M. R., L. C. Chaudhary, C. S. Bestwick, A. J. Richardson, N. McKain, T. R. Larson, I. A. Graham, and R. J. Wallace. 2010. Toxicity of unsaturated fatty acids to the biohydrogenating ruminal bacterium, Butyrivibrio fibrisolvens. BMC Microbiol. 10:52-61.

Maia, M. R., L. C. Chaudhary, L. Figueres, and R. J. Wallace. 2007. Metabolism of polyunsaturated fatty acids and their toxicity to the microflora of the rumen. Antonie Van Leeuwenhoek 91:303-314.

Paillard, D., N. McKain, L. C. Chaudhary, N. D. Walker, F. Pizette, I. Koppova, N. R. McEwan, J. Kopecny, P. E. Vercoe, P. Louis, and R. J. Wallace. 2007. Relation between phylogenetic position, lipid metabolism and butyrate production by different Butyrivibrio-like bacteria from the rumen. Antonie Van Leeuwenhoek 91:417-422.

Pitta, D. W., W. Pinchak, S. Dowd, J. Osterstock, V. Gontcharova, E. Youn, K. Dorton, I. Yoon, B. Min, J. D. Fulford, T. Wickersham, and D. Malinowski. 2010. Rumen bacterial diversity dynamics associated with changing from bermudagrass hay to grazed winter wheat diets. Microb. Ecol. 59:511-522.

Pope, P. B., A. K. MacKenzie, I. Gregor, W. Smith, M. A. Sundset, A. C. McHardy, M. Morrison, and V. G. H. Eijsink. 2012. Metagenomics of the Svalbard reindeer rumen microbiome reveals abundance of polysaccharide utilization loci. PLoS ONE 7:e38571.

Popova, M., C. Martin, M. Eugène, M. M. Mialon, M. Doreau, and D. P. Morgavi. 2011. Effect of fibre- and starch-rich finishing diets on methanogenic Archaea diversity and activity in the rumen of feedlot bulls. Anim. Feed Sci. Technol. 167:113-121.

Shingfield, K. J., P. Kairenius, A. Äröla, D. Paillard, S. Muetzel, S. Ahvenjärvi, A. Vanhatalo, P. Huhtanen, V. Toivonen, J. M. Griinari, and R. J. Wallace. 2012. Dietary fish oil supplements modify ruminal biohydrogenation, alter the flow of fatty acids at the omasum, and induce changes in the ruminal Butyrivibrio population in lactating cows. J. Nutr. 142:1437-1448.

Stevenson, D. M., and P. J. Weimer. 2007. Dominance of Prevotella and low abundance of classical ruminal bacterial species in the bovine rumen revealed by relative quantification real-time PCR. Appl. Microbiol. Biotechnol. 75:165-174.

Stewart, C. S., H. J. Flint, and M. P. Bryant. 1997. The rumen bacteria. Pages 10-72 in The Rumen Microbial Ecosystem. P.N. Hobson and C.S. Stewart, ed. Chapman \& Hall, London, UK.

Tokoyama, M. T., and C. L. Davis. 1970. Hydrogenation of linoleic acid by Borrelia B25 and Succinivibrio dextrinosolvens. Fed. Proc. 29:A691.

Toral, P. G., A. Belenguer, K. J. Shingfield, G. Hervás, V. Toivonen, and P. Frutos. 2012. Fatty acid composition and bacterial community changes in the rumen fluid of lactating sheep fed sunflower oil plus incremental levels of marine algae. J. Dairy Sci. 95:794-806.

van Gylswyk, N. O. 1990. Enumeration and presumptive identification of some functional groups of bacteria in the rumen of dairy cows fed grass silage-based diets. FEMS Microbiol. Lett. 73:243-261.

Wu, S., R. L. Baldwin, W. Li, C. Li, E. E. Connor, and R. W. Li. 2012. The bacterial community composition of the bovine rumen detected using pyrosequencing of $16 \mathrm{~s}$ rRNA genes. Metagenomics $1: 235571$.

Zened, A., S. Combes, L. Cauquil, J. Mariette, C. Klopp, O. Bouchez, A. Troegeler-Meynadier, and F. Enjalbert. 2013. Microbial ecology of the rumen evaluated by 454 GS FLX pyrosequencing is affected by starch and oil supplementation of diets. FEMS Microbiol. Ecol. 83:504-514. 